\title{
Advancement in the treatment of diminished ovarian reserve by traditional Chinese and Western medicine (Review)
}

\author{
CHEN ZHANG $^{1}$ and XIA XU ${ }^{2}$ \\ ${ }^{1}$ Nanjing University of Chinese Medicine, Nanjing 210000; ${ }^{2}$ Xuzhou City Hospital of \\ Traditional Chinese Medicine, Xuzhou, Jiangsu 221000, P.R. China
}

Received November 9, 2015; Accepted January 22, 2016

DOI: $10.3892 /$ etm.2016.3025

\begin{abstract}
Diminished ovarian reserve (DOR) refers to the decreased production of mature ova in ovarium and decreased quality of oocyte, leading to deficiency of the female sex hormone and decreased fertility. DOR can also develop into premature ovarian failure, which affects female life quality and constitutes an important cause of female infertility. A fast lifestyle, environmental deterioration and accumulated understanding of this disease, have led to an increase in the incidence of DOR. Therefore, reasonable and effective treatment for DOR is particularly important to improve ovarian function and female life quality as well as reduce infertility. This mini review provides recent updates regarding DOR and a summary of advancements in the treatment of DOR using Chinese and Western Medicine.
\end{abstract}

\section{Contents}

1. Introduction

2. Traditional Chinese medicine treatment

3. Western medicine treatment

4. Integrative medicine

\section{Introduction}

Diminshed ovarian reserve (DOR) is a condition in which women between the ages of 16 and 40 experience impaired ovarian function, whereby the ovarian reserve is decreased or the quality of oocyte is reduced (1). Clinical manifestations of DOR patients include oligomenorrhea, hypomenorrhea, amenorrhea and infertility (1). In most cases, DOR is accompanied by soreness and weakness of waist and knees, hot flashes and

Correspondence to: $\mathrm{Dr} \mathrm{Xia} \mathrm{Xu}$, Xuzhou City Hospital of Traditional Chinese Medicine, 169 Zhongshannan Road, Xuzhou, Jiangsu 221000, P.R. China

E-mail: xuxia_9@163.com

Key words: diminished ovarian reserve, integrative medicine sweating, insomnia and distraction (2). DOR is not identified in traditional Chinese medicine (TCM), but is classified as a symptom of delayed menorrhea, oligomenorrhea, amenorrhea, irregular menstrual cycle and infertility (3). Currently, there are many therapies for DOR. TCM treatment for DOR includes syndrome differentiation, specific prescriptions and medications, as well as TCM periodic therapy and acupuncture therapy (4). Western medicine primarily utilizes hormone replacement therapy (HRT), oral contraceptives (OCP), dehydroepiandrosterone (DHEA), ovulation induction therapy, and assisted reproductive technique (ART) (4).

The present study reviewed advancements in the treatment of DOR by TCM and Western medicine, and provided scientific evidence for the study and treatment of DOR.

\section{Traditional Chinese medicine treatment}

TCM treatment based on syndrome differentiation. $\mathrm{Xu}$ and Tan (1) studied TCM treatment of DOR based on syndrome differentiation, which included nephrasthenia syndrome, Guishao and Dihuang decoction, blood-stasis syndrome, Xuefu Zhuyu decoction, syndrome of upper hyperactivity of liver yang, Tianma Gouteng drink, cardiac and renal failure, Qing Xin Zi Shen decoction, syndrome of yang deficiency of spleen and kidney, and Jian Gu decoction. Hang (5) used treatment based on syndrome differentiation with Yu Lin Fang plus or minus nourishing blood and replenishing essence, reinforcing kidney and Tiao Chong (5). In patients with stagnation of QI due to liver depression, identification of smooth liver, and regulation of QI and menstruation is important. In patients with stagnation of blood stasis, the blood circulation is promoted to remove meridian obstruction and eliminate stasis syndrome (5). Dampness in the lungs leading to phlegm in patients should be eliminated, cold-dampness should be warming Yang to dispel the cold in order to dissipate the cold and remove the dampness, thereby clear away heat and toxic material for damp heat patients (5). These forms of treatment were of good efficacy and worthy of clinical application. Li (6) studied the application of clinical TCM treatment for delayed menorrhea (DOR) including blood deficiency syndrome involving invigorating kidney and boosting essence, nourishing blood and the promotion of blood circulation; kidney deficiency and liver stagnation syndrome, invigorating kidney and the promotion of blood circulation, soothing the liver and 
regulating QI; syndrome of Yang deficiency of spleen and kidney, warming kidney and strengthening spleen, reinforcing QI and nourishing blood; and syndrome of Yin deficiency and blood dryness, nourishing kidney and dryness, nourishing blood and promoting blood circulation.

Specific prescriptions and medications. Shi et al (7) observed 40 DOR patients with weak spleen and kidney. These patients were administered with Zishen Yutai pill after 5 days of menstruation. At 3 months after treatment, the symptoms of soreness and weakness of waist and knees, dizziness and tinnitus, as well as insomnia and distraction significantly improved. The levels of serum follicle-stimulating hormone (FSH) and luteinizing hormone $(\mathrm{LH})$ decreased $(\mathrm{P}<0.05)$, while the levels of anti-Müllerian hormone $(\mathrm{AMH})$ and inhibin increased $(\mathrm{P}<0.05)(6)$. Meng and Fan $(8)$ provided a follicle-stimulating therapy for detoxifying kidney and removing blood stasis in 98 patients with DOR and infertility. The symptoms were effectively resolved, with a significantly decreased FSH level and increased ovulation rate as well as pregnancy rate. Zhang (9) treated DOR patients with black turtle therapy for three menstrual cycles. Black turtle therapy may significantly improve clinical symptoms, increase ovarian antral follicles and serum E2 level, decrease the levels of FSH and LH, and improve ovarian reserve, as well as the successful rate of assisted reproductive technology, preventing and delaying premature ovarian failure (POF) (9).

TCM periodic therapy. Huang et al (10) treated 78 DOR patients by detoxifying kidney and regulating menstruation in combination with Western medicine for 3 months continuously, i.e., nourishing kidney Yin in the follicular phase, nourishing Yin and Yang in the ovulatory phase, warming kidney and invigorating Yang in the luteal phase, and promoting blood flow for the regulation of the menstruation cycle. Detoxifying kidney and regulating menstruation significantly improved ovarian reserve, the serum levels of FSH, LH and the FSH/LH ratio were decreased $(\mathrm{P}<0.05)$ and the antral follicle count was increased $(\mathrm{P}<0.05)(10)$. Meng et al $(11)$ treated $100 \mathrm{DOR}$ patients with TCM periodic therapy, i.e., Yi Mu Dang Yang Fang in the follicular phase, nourishing kidney yang with Tiaozhutang in the luteal phase, regulating Qi and blood with ovulation decoction in the ovulatory period. After 3 months of treatment, the FSH level and FSH/LH ratio were decreased and E2 level was increased, with a total efficiency of 95\% (11). Ruan (12) considered the physiological characteristics of each phase in the menstrual cycle, and used Zuogui pill as the basic formula, adding $20 \mathrm{~g}$ Codonopsis pilosula, $20 \mathrm{~g}$ Poria cocos, 10 g Rhizoma Anemarrhenae, 10 g Rhizoma Cyperi in the follicular phase, and $10 \mathrm{~g}$ Cassia twig, $10 \mathrm{~g}$ Morinda officinalis, $10 \mathrm{~g}$ Angelica sinensis, and $10 \mathrm{~g}$ Amomi Cardamomi Fructus in the luteal phase (13). The symptoms of delayed menstrual cycle, hypomenorrhea, soreness and weakness of waist and knees, insomnia and distraction, hot flashes and perspiration in 37 DOR patients were significantly improved (total efficiency 91.89\%), and the levels of FSH, FSH/LH and E2 were significantly decreased $(\mathrm{P}<0.05)$.

Acupuncture therapy. Zhang et al (13) examined the efficacy of acupuncture in combination with TCM for soothing the liver, nourishing kidney and promotion of blood circulation in 50 DOR patients. Acupuncture was performed at the Tsusanli, Guanyuan, Sanyinjiao, Diji, Qihai, Zigong, Shenshu, Ganshu, Pishu acupoints, as well as the Yinlingquan and Fenglong acupoints for phlegm stagnation patients; Hegu, Xuehai and Taichong acupoints for patients of Qi stagnancy and blood stasis; and Mingmen and Yaoyangkuan acupoints for patients with cold coagulation. In addition, Xiaoyao pill and Zuigui pill were added. After three courses of treatment, DOR patients achieved significant efficacy with acupuncture and TCM for soothing liver, nourishing kidney and promotion of blood circulation (13). Zhang (14) treated DOR (deficiency of kidney yin) with Zuigui pill and auricular-plaster therapy, which significantly improved the clinical symptoms, decreased the levels of FSH, LH and E2, and successfully increased the pregnancy rate.

\section{Western medicine treatment}

Hormone replacement therapy. HRT is the most commonly used therapy for DOR. Li and Chen (15) treated 78 DOR patients with sequential estrogen and progestogen therapy. After three courses of treatment, the baseline levels of FSH, $\mathrm{LH}$ and the FSH/LH ratio were significantly decreased $(\mathrm{P}<0.05)$, and the ovulation rate was significantly increased $(\mathrm{P}<0.05)$. However, the relative contraindications and side reactions limited the clinical applications of HRT, increasing the incidence of endometrial cancer, breast cancer and cardiovascular diseases.

Oral contraceptives. OCP such as desogestrel and ethinylestradiol (Marvelon) inhibit the hypothalamic-pituitary-ovarian axis in a feedback manner, decrease the levels of FSH and E2, improve ovarian function and increase pregnancy rate. Pretreatment with OCP prior to ovulation induction may regulate the menstrual cycle in the patients with decreased ovarian function, increase synchronicity of follicular development, decrease cycle cancellation rate and obtained better ovulation induction. However, pretreatment with OCP may inhibit the increase of early endogenous FSH, gradual follicular development, prolonging the time of medication (16). However, a large number of studies are required to evaluate the impact of OCP on DOR.

Dehydroepiandrosterone. Previous findings identified that androgen was able to increase follicular sensitivity to FSH through action on the androgen receptor (17). DHEA was an androgen secreted by adrenal glands, the central nervous system and ovarian theca cells. Zhang (18) evaluated the effect of DHEA on the ovarian function of DOR patients and main adverse reactions of DHEA. Three months after DHEA treatment ( $50 \mathrm{mg} /$ day), the serum level of FSH was decreased, the $\mathrm{AMH}$ level was increased, the antral follicle count was increased and the ovarian reserve was improved. However, the clinical efficacy of DHEA remains to be clarified.

Ovulation induction therapy. A number of ovulation induction therapies are available for DOR patients, including induced ovulation and superovulation. Induced ovulation is regarded as the induction of $\geq 1$ follicular growth by drugs or surgery 
in rare-ovulation or anovulation patients (19). Superovulation is known as controlled ovarian hyper $(\mathrm{COH})$ stimulation, is utilized in the controlled induction of multiple follicular development and maturation in in vitro fertilization (IVF) (20). Commonly used ovulation induction therapies included long-term gonadotropin-releasing hormone agonist (GnRH-A) therapy, short-term GnRH-A therapy, antagonists therapy and microstimulation. It was reported that microstimulation achieved improved clinical outcome in the patients with poor ovarian reserve (21).

Assisted reproductive technique. Assisted reproductive technique (ART) successfully resolved fertility issues in many women and has been extensively used in the treatment of infertility, especially IVF-embryo transfer (ET) (21). Obtaining multiple quality eggs and sufficient quality embryos by $\mathrm{COH}$ was critical for successful IVF-ET. Geng et al examined different forms of stimulatation of ovulation solution in 87 patients who underwent IVF - ET owing to ovarian low reactivity. The microstimulation was found to have a higher cost-benefit ratio, and was deemed significant and applicable in the patients with ovarian low reactivity (22).

Ovum donation. Ovum donation is a new technology in the treatment of infertility and may be useful to DOR patients. However, the clinical application of ovum donation has been limited owing to ethical, moral, and legal issues (23).

Ovarian transplantation. Ovarian transplantation may recover the endocrine and reproductive function of DOR patients. Along with the development of animal- and human-assisted reproductive technology, particularly ovarian cryopreservation, oocyte in vitro maturation and fertilization have led to the improvement of the technology, technology combined with ovarian transplantation is considered a breakthrough, and is rapidly leading to the preservation and perpetuation of an important technology for female reproductive ability (24). The development of ovarian transplantation, ovary autologous orthotopic or heterotopic transplantation, and ovarian xenograft have led to clinical application. However, various problems for ovarian transplantation, such as ischemia, injury of transplanted ovary by cryopreservation and immunological rejection have been previously identified.

\section{Integrative medicine}

Zhang et al (25) analyzed 35 infertile DOR patients who became pregnant following Integrative Medicine treatment. The study demonstrated that estradiol valerate tablets+ethinyl+TCM for detoxifying the kidney and promotion of the blood circulation may improve ovarian function, increase pregnancy rate. The serum levels of FSH, E2 $(\mathrm{P}<0.05)$ and the FSH/LH ratio $(\mathrm{P}<0.05)$ were higher after treatment. Liu (26) observed the clinical efficacy of nourishing kidney decoction and Western medicine in the treatment of oligomenorrhea due to DOR. At 3 months after treatment, menstruation was significantly improved, the levels of FSH and LH were significantly decreased, and E2 level was significantly increased, with a total efficiency of $93.33 \%$. Zhang and $\mathrm{Li}$ (27) provided sequential artificial cycle therapy (estradiol valerate+progesterone injection) in 46 infertile DOR patients prior to ovulation induction and TCM Tiaojing Cuyun pill for 3 months. Pretreatment resulted in the decreased FSH level and FSH/LH ratio, increased E2 level, with a pregnancy rate of $35 \%$ and an ovulation rate of $54 \%$.

TCM is unique to improvement of ovarian reserve and increasing the ovarian responsiveness to gonadotrophin and ovarian sex hormone level due to the multi-system and multi-step regulatory functions. In addition, TCM has few side effects, and extensive clinical applications, although medication time and efficacy are gradual. The long-term efficacy of Western medicine in the treatment of DOR is unsatisfactory, with many contraindications and adverse reactions. The treatment of DOR with integrative medicine achieved good clinical efficacy. However, there were no uniform diagnostic criteria, therapeutic regime and evaluation criteria for DOR. Therefore, further investigation is required to develop a safe and efficacious therapy to improve ovarian reserve, prevent POF and treat infertility with DOR.

\section{References}

1. Cao Z-Y: Obstetrics and Gynaecology (2nd edition). People's Medical Publishing House, Beijing, 2004 (In Chinese).

2. Zhu L, Chen CX and Yin WW: A study on the pathogenesis of 72 patients with diminished ovarian reserve. Chin J Tradit Chin Med 11: 1233-1234, 2012 (In Chinese).

3. Xu X and Tan Y: Treatment of diminished ovarian reserve by Traditional Chinese Medicine syndrome differentiation. Univ Tradit Chin Med 3: 325-327, 2010 (In Chinese).

4. Tang WL, Hu YH and He XH: Research progress of traditional Chinese medicine and Western Medicine on the decline of ovarian reserve. J Shanxi Med 15: 1797-1799, 2014 (In Chinese).

5. Hang Y: The experience of He Jialin in the treatment of decreased ovarian reserve. Proc Zhejiang Univ Tradit Chin Med 38: 1064-1065, 2014 (In Chinese).

6. Li X: Clinical application of TCM approaches in delayed menorrhea (Diminished ovarian reserve). Chengdu Univ Tradit Chin Med: 2014 (In Chinese).

7. Shi Y, Yang S and Tao L: Clinical observation of Zishen Butai pill in the treatment of diminished ovarian reserve with weak spleen and kidney. Proc Shandong Univ Tradit Chin Med 37: 292-294, 2013.

8. Meng X and Fan Y: Clinical observation of the pre-treatment in 98 infertile DOR patients. Matern Child Health Care China 27: 2973-2975, 2012.

9. Zhang J: Clinical study of Black turtle in the treatment of diminished ovarian reserve. Nanjing Univ Chin Med: 2013 (In Chinese).

10. Huang X, Liang S and Lu W: Clinical observation of tonifying kidney and regulating menstruation combined with Western medicine in the treatment of DOR. Chin J Integr Med 34: 688-690, 2014 (In Chinese).

11. Meng Q, Fu Y and Cao B: The effect of TCM periodic treatment on ovarian reserve. J Tradit Chin Med 31: 644-645, 2011 (In Chinese).

12. Ruan H: Clinical observation of TCM periodic treatment in DOR patients. Guangzhou Univ Chin Med: 2011 (In Chinese).

13. Zhang Y, Fang Y and Li L: Clinical observation of the effect of acupuncture and drugs on ovarian reserve. Sichuan Tradit Chin Med 28: 103-104, 2010 (In Chinese).

14. Zhang M: Clinical observation of Zuigui pill and auricular plaster in the treatment of DOR patients with deficiency of kidney-Yin. Guangzhou Univ Chin Med: 2012.

15. Li Y and Chen C: Clinical observation of sequential therapy with estrogen and progestogen in the treatment of diminished ovarian reserve. Guide Chin Med 10: 113-114, 2012 (In Chinese).

16. Qian Q, Sun L and Wang X: The effect of oral contraceptives in the short-term ovulation induction by IVF-ET. Chin J Birth Health Hered 19: 102-104, 2011. 
17. Nielsen ME, Rasmussen IA, Kristensen SG, Christensen ST, Møllgård K, Wreford Andersen E, Byskov AG and Yding Andersen C: In human granulosa cells from small antral follicles, androgen receptor mRNA and androgen levels in follicular fluid correlate with FSH receptor mRNA. Mol Hum Reprod 17: 63-70, 2011 (In Chinese).

18. Zhang D: Clinical effect of dehydroepiandrosterone in diminished ovarian reserve. Union School Clin Med: 2013.

19. Rugh R: Induced ovulation and artificial fertilization inthe frog. Biolog Bull 66: 22-23, 1934.

20. Broer SL, Dólleman M, Opmeer BC, Fauser BC, Mol BW and Broekmans FJ: AMH and AFC as predictors of excessive response in controlledovarian hyperstimulation: a meta-analysis. Hum Reprod Update 17: 46-54, 2010.

21. Revelli A, Casano S, Salvagno F and Delle Piane L: Milder is better? Advantages and disadvantages of 'mild' ovarian stimulation for human in vitro fertilization. Reprod Biol Endocrinol 9 : 25, 2011 (In Chinese)
22. Geng JX, Zhang SD and Zhang CL: Application of different ovulation induction programs in patients with low ovarian response. Reprod Contracept 30: 837-840, 2010.

23. Sauer MV: Ethics and reproductive medicine. Preface. Semin Reprod Med 28: 277-280, 2010.

24. Peng N, Yuan A and Zou H: Ovary transplantation and its applications. Adv Vet Med 32: 99-103, 2011.

25. Zhang L, Zhao F and Wang G: The efficacy of Integrative Medicine in the treatment of infertile DOR patients. Matern Child Health Care China 26: 4293-4295, 2014.

26. Liu L: Clinical observation of nourishing kidney decoction and Western medicine in the treatment of oligomenorrhea due to DOR. Heilongjiang Univ Tradit Chin Med: 2012

27. Zhang T and Li S: Observation of 46 infertile DOR patients receiving pre-treatment. Clin Med (Northfield IL) 30: 78-79, 2010. 